\title{
G994T polymorphism in exon 9 of plasma platelet-activating factor acetylhydrolase gene and lung ultrasound score as prognostic markers in evaluating the outcome of acute respiratory distress syndrome
}

\author{
WANG LV ${ }^{1}$, SHENGNAN WANG ${ }^{2}$, LEI WANG ${ }^{1}$, ZHANG WU $^{1}$, \\ YING YING JIANG $^{1}$, XINGUO CHEN $^{1}$ and RENXIAN GAO ${ }^{1}$ \\ Departments of ${ }^{1}$ Emergency and ${ }^{2}$ Rheumatology and Immunology, Wenzhou People's Hospital, \\ Wenzhou, Zhejiang 325000, P.R. China
}

Received August 21, 2017; Accepted November 7, 2018

DOI: $10.3892 / \mathrm{etm} .2019 .7281$

\begin{abstract}
The present study aimed to discover potential biomarkers for predicting the prognosis of acute respiratory distress syndrome (ARDS) in conjunction with lung ultrasound (LUS). Blood samples from 112 ARDS patients were collected to compare their partial oxygen pressure $\left(\mathrm{PaO}_{2}\right)$ /fraction of inspired oxygen $\left(\mathrm{FiO}_{2}\right)$, positive end-expiratory pressure (PEEP), lactic acid, sequential organ failure assessment (SOFA) score, clinical pulmonary infection score (CPIS) and APACHE II score. Kaplan-Meier plots and the log-rank test were performed to analyse the association between the platelet-activating factor acetylhydrolase (PAFAH) G994T polymorphism and the outcome of ARDS regarding mortality. A negative correlation between the LUS score and $\mathrm{PaO}_{2} / \mathrm{FiO}_{2}$, PEEP and lactic acid, as well as with the SOFA, CPIS and APACHE II score was confirmed with correlation coefficients of $-0.493,-0.548,-0.642,-0.598$, -0.566 and -0.567 , respectively (all $\mathrm{P}<0.05$ ). The activity of PAFAH and high-density lipoprotein-PAFAH in the serum collected from subjects of the GG genotype was similar to that in subjects of the GT genotype, but the low-density lipoprotein-PAFAH activity in the serum collected from GG subjects was significantly higher than that in GT subjects. An evident reduction in the PEEP, level of lactic acid, as well as the SOFA, CPIS and APACHE II score was observed in GG subjects, accompanied by a significantly increased $\mathrm{PaO}_{2} / \mathrm{FiO}_{2}$. Kaplan-Meier analysis indicated that subjects
\end{abstract}

Correspondence to: Dr Renxian Gao, Department of Emergency, Wenzhou People's Hospital, 57 Canghou Street, Wenzhou, Zhejiang 325000, P.R. China

E-mail: lusards@yeah.net

Key words: G994T polymorphism, platelet-activating factor acetylhydrolase, acute respiratory distress syndrome, lung ultrasound, inflammation with a high LUS score had a significantly higher survival rate than those with a low LUS score, and the mortality risk for GG subjects was significantly lower than that for GT subjects. Finally, among all groups (genotype and LUS groups), GG subjects with a high LUS score had the lowest mortality risk, whereas GT subjects with a low LUS score had the highest mortality risk. In addition, the survival rate of GT subjects with a high LUS score was higher than that of GG subjects with a low LUS score. In conclusion, the combination of the LUS score and the G994T polymorphism in exon 9 of the PAFAH gene may be used as a potential prognostic marker for ARDS.

\section{Introduction}

Acute respiratory distress syndrome (ARDS) is a disorder comprising pulmonary inflammation induced by direct or indirect damage to the lungs (1). To date, management of ARDS has failed to decrease the mortality rate of ARDS to $<30 \%$, primarily due to an insufficient understanding of the pathophysiology of ARDS. The stimulation of platelets in the lung microvasculature and the sequestration of leukocytes, particularly neutrophils, appear to have critical roles in the pathophysiology of ARDS, resulting in the formation of microthrombus and blockage of blood circulation (2-4).

ARDS is common in critically ill patients. Of those patients receiving mechanical ventilation in the intensive care unit (ICU), 19\% suffer from ARDS, which is associated with refractory hypoxemia and an elevated level of extravascular lung water (EVLW), and has a mortality rate ranging from 32 to $65 \%$ (5). In the early stage of ARDS, lung ultrasound (LUS) is performed to locate pulmonary edema, and 'B-lines' in LUS indicate a reduced level of lung aeration, likely triggered by an elevated level of EVLW (6). Based on the pulmonary ultrasonography in patients with ARDS, certain studies have recommended the use of LUS to measure EVLW semi-quantitatively (7). It has been demonstrated that, as a user-friendly, simple, cost-efficient and non-invasive bedside test, LUS was closely associated with the prognosis and 
mortality risk of ARDS (8), and may thus be used to determine the best treatment scheme for ARDS patients (8).

The level of platelet-activating factors (PAF), a family of pro-inflammatory lipids present in newborns with an immature immune system, is increased in infants affected by inflammatory conditions, including necrotizing enterocolitis (9). Earlier studies in rodents and humans revealed that PAF acetylhydrolase (PAFAH), which is present in breast milk and is able to degrade PAF, probably has a pivotal role in inhibiting the inflammatory effect of PAF (10).

Pro-inflammatory PAF is one of the strongest neutrophil activators and functions via a specific PAF receptor, which belongs to the family of seven transmembrane G-protein-coupled receptors (11). Deletion of the acetyl group at the sn- 2 site of PAF by PAFAH results in the inactivation and degradation of PAF (12). Circulating in the blood, PAFAH binds to and degrades oxidized phospholipids and PAF that are biologically active (13). Earlier studies demonstrated that critically ill patients with sepsis had a reduced activity of plasma PAFAH when compared with that in healthy subjects (14). In addition, changes in the activity of plasma PAFAH may affect the severity of asthma (15). Furthermore, a correlation between plasma PAFAH activity, allelic alterations and the severity of ARDS has been demonstrated (11).

A point mutation at the nucleotide site 994 in exon 9 [single-nucleotide polymorphism (SNP) ID, rs16874954] leads to a G994 $\rightarrow$ T mutation in the PAFAH gene, resulting in a Val279 $\rightarrow$ Phe substitution in the mature protein (V279F), which is located in the catalytic domain of the enzyme, thus reducing its catalytic activity. In Japanese families with a deficiency in plasma PAFAH activity, the enzymatic activity of PAFAH is completely abolished in subjects harbouring the TT homozygotes, whereas a decreased PAFAH activity is observed in subjects harbouring the GT homozygotes (16-18).

Since the LUS score and an SNP in PAFAH have been demonstrated to be associated with the prognosis of ARDS (16-18), the present study evaluated the potential of assessing different PAFAH variants in conjunction with the LUS score to predict the prognosis of ARDS.

\section{Materials and methods}

Patients and samples. Blood samples were collected from 112 patients diagnosed with ARDS. All subjects were recruited at Wenzhou People's Hospital (Wenzhou, China) within $72 \mathrm{~h}$ of their ARDS diagnosis and none of them suffered from left ventricular failure. Baseline PAFAH measurements were performed using blood samples collected prior to any treatment. The partial oxygen pressure $\left(\mathrm{PaO}_{2}\right)$ /fraction of inspired oxygen $\left(\mathrm{FiO}_{2}\right)$, positive end-expiratory pressure (PEEP) and lactic acid levels were recorded for each patient. The protocol of the present study was approved by the Ethics Committee of Wenzhou People's Hospital (Wenzhou, China) and written informed consent was obtained from all participants prior to the study. The study was performed according to the Declaration of Helsinki.

Genotyping. Genomic DNA was extracted from blood samples and subjected to amplification by polymerase chain reaction (PCR). Electrophoresis on $2.5 \%$ agarose gels was performed to analyse the PCR products and a restriction analysis was performed to determine the PAFAH G994T genotypes. The TaqMan method (Roche, Basel, Switzerland) was utilized to analyse the SNPs (19).

PAFAH activity measurement. A Human Platelet-activating Factor Acetylhydrolase ELISA kit (cat. no. E0699Hu; Shanghai Korain Biotech, Co., Ltd., Shanghai, China) was utilized to determine PAFAH activity, including total PAHAH activity, low-density lipoprotein-PAFAH (L-PAFAH) and high-density lipoprotein-PAFAH (H-PAFAH). Blood samples collected from patients with ARDS within $72 \mathrm{~h}$ of their diagnosis were utilized.

APACHE II, clinical pulmonary infection score (CPIS) and sequential organ failure assessment (SOFA) scoring. The APACHE II (20), CPIS (21) and SOFA (22) score were evaluated as described previously.

LUS scoring. An M-Turbo ultrasound machine (FUJIFILM SonoSite, Bothell, WA, USA) equipped with a 2- to $5-\mathrm{MHz}$ curved array probe was used to perform all tests. Prone position lung ultrasound examination was performed immediately after the prone positioning was started (H0), as well as at 3 and $6 \mathrm{~h}$ after the initialization of prone positioning ( $\mathrm{H} 3$ and $\mathrm{H} 6$, respectively). The points of examination were located on the posterior axillary line, the scapular line and the paravertebral line. These three lines served as body markers to divide one side of the back into three regions, and each region was subsequently divided into 3 areas of equal size. Therefore, a total of 9 examination areas were obtained on each subject, and 8 points (excluding the point covered by the scapular bone) were analysed on each side of the back, with a total of 16 points being analysed on each subject. Sonographic signs of lung aeration were divided into four groups as follows: i) Consolidation (C) indicated by a tissue pattern characterized as dynamic air bronchograms; ii) severe loss of lung aeration (B2) indicated by coalescent B lines; iii) moderate loss of lung aeration indicated by multiple spaced B lines; iv) normal pattern $(\mathrm{N})$ indicated by lung sliding with isolated B lines $(<3)$ or A lines. During the analysis, the points in a particular region were scored based on the worst ultrasound pattern, and the scores of all points were added. By definition, the aeration score variation was the difference between scores at various time-points (H6 vs. $\mathrm{H} 3$ and $\mathrm{H} 3$ vs. H0). All LUS video recordings were scored and evaluated by two independent ICU physicians in a blinded manner.

Statistical analysis. All values are expressed as the mean \pm standard deviation. Independent-samples t-tests were utilized to evaluate differences between the groups. $\chi^{2}$ analyses were performed to evaluate the deviations of PAFAH G994T genotype distributions from the Hardy-Weinberg equilibrium, and to calculate the frequencies of genotypes or alleles among different groups. Logistic regression and $\chi^{2}$ analysis were performed to calculate $95 \%$ confidence intervals and odds ratios, so as to determine the risk associated with the $\mathrm{T}$ allele. Kaplan-Meier plots and the log-rank test were performed to analyse the association between PAFAH G994T polymorphism and the outcome of ARDS patient mortality. A Pearson's analysis was performed to assess correlation between PAFAH G994T genotype and the activity of PAFAH. 

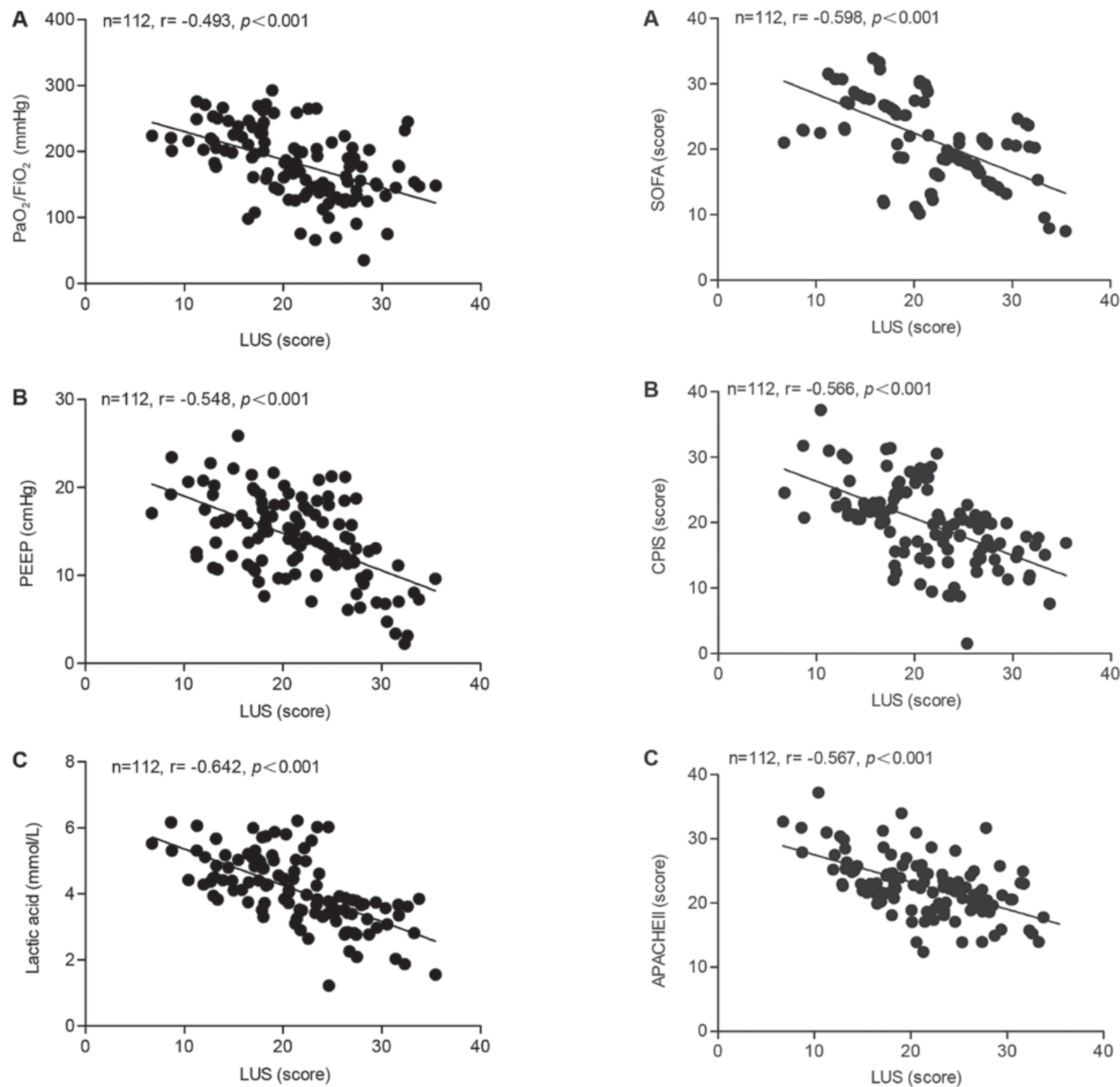

Figure 1. Pearson analysis of the correlation between LUS score and $\mathrm{PaO}_{2} / \mathrm{FiO}_{2}$, PEEP or lactic acid in patients with acute respiratory distress syndrome. (A) The correlation between LUS score and $\mathrm{PaO}_{2} / \mathrm{FiO}_{2}$ was negative, and the correlation coefficient was -0.493. (B) A negative correlation between LUS score and PEEP was confirmed with a correlation coefficient of -0.548 . (C) A negative correlation between LUS and lactic acid was confirmed with a correlation coefficient of -0.642 . LUS, lung ultrasound; $\mathrm{PaO}_{2} / \mathrm{FiO}_{2}$, partial oxygen pressure/fraction of inspired oxygen; PEEP, positive end-expiratory pressure.

$\mathrm{P}<0.05$ was considered to indicate a statistically significant difference. SPSS version 17.0 (SPSS, Inc., Chicago, IL, USA) was used to perform all statistical analyses.

\section{Results}

The LUS score is correlated with the partial oxygen pressure $\left(\mathrm{PaO}_{2}\right) /$ fraction of inspired oxygen $\left(\mathrm{FiO}_{2}\right)$, positive end-expiratory pressure (PEEP) and lactic acid levels. The correlation between the LUS score and the $\mathrm{PaO}_{2} / \mathrm{FiO}_{2}$ ratio, PEEP and lactic acid was determined using blood samples collected from 112 ARDS patients. A significant negative

correlation was identified between the LUS score and the $\mathrm{PaO}_{2} / \mathrm{FiO}_{2}$ ratio (Fig. 1A), PEEP (Fig. 1B) and lactic acid (Fig. 1C), with correlation coefficients of $-0.493,-0.548$ and -0.642 , respectively. Furthermore, the correlation between the LUS and the SOFA, CPIS or APACHE II score was calculated. As presented in Fig. 2, negative correlation coefficients of $-0.598,-0.566$ and -0.567 were determined for the correlation between the LUS and the SOFA (Fig. 2A), the CPIS (Fig. 2B) and the APACHE II score (Fig. 2C), respectively.

Association between serum PAFAH activity and PAHAF G994T genotype. In the next step, the subjects were genotyped 

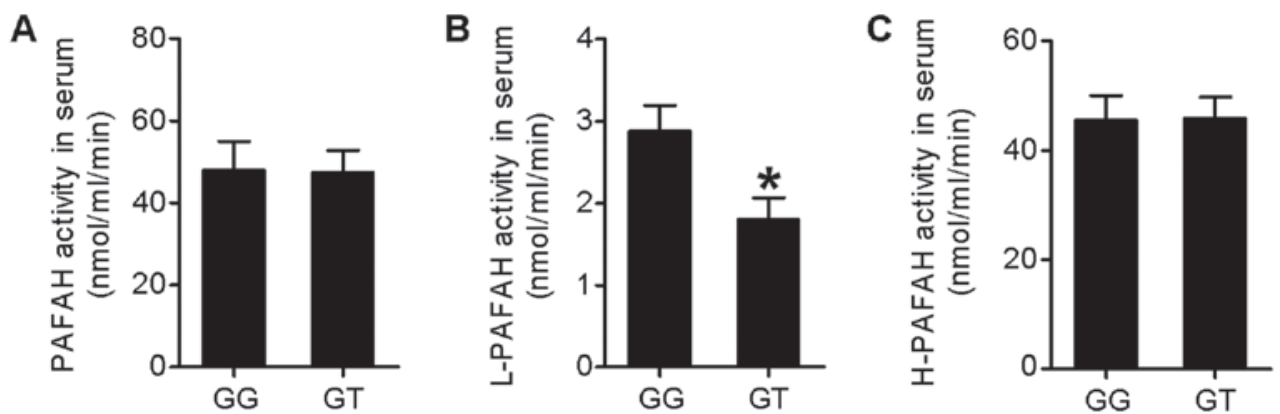

Figure 3. Association between serum PAFAH activity and PAFAH G994T genotype in patients with acute respiratory distress syndrome. (A) The PAFAH activity in subjects of the GG genotype was not significantly different from that in subjects of the GT genotype. (B) L-PAFAH activity in the serum of patients of the GG genotype was significantly higher than that in patients of the GT genotype. (C) H-PAFAH activity in the GG group was comparable with that in the GT group. "P<0.05 vs. GG. PAFAH, platelet-activating factor acetylhydrolase; L, low-density lipoprotein; H, high-density lipoprotein.
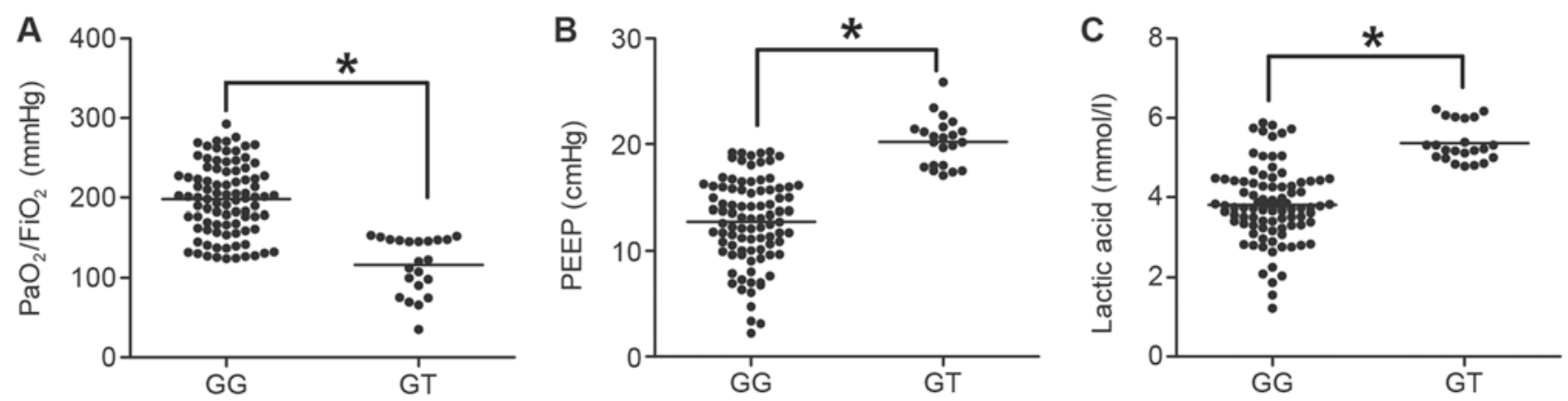

Figure 4. The platelet-activating factor acetylhydrolase G994T polymorphism is associated with $\mathrm{PaO}_{2} / \mathrm{FiO}_{2}, \mathrm{PEEP}$ and lactic acid in patients with acute respiratory distress syndrome. (A) $\mathrm{PaO}_{2} / \mathrm{FiO}_{2}$ ratio in the GG group was higher than that in the GT group. (B) A lower PEEP was observed in the GG group compared with that in the GT group. (C) The GG group had a lower level of lactic acid than the GT group. ${ }^{.} \mathrm{P}<0.05$. $\mathrm{PaO}_{2} / \mathrm{FiO}_{2}$, partial oxygen pressure/fraction of inspired oxygen; PEEP, positive end-expiratory pressure.

for the PAHAF G994T polymorphism, with 90 subjects being genotyped as GG and 22 subjects being genotyped as GT (none of the subjects was genotyped as TT). PAFAH activities, including total activity of PAHAH, low-density L-PAFAH and H-PAFAH, were measured using the blood samples collected from ARDS subjects within $72 \mathrm{~h}$ of their diagnosis. As presented in Fig. 3, the activity of total PAFAH and H-PAFAH in the serum exhibited no difference between subjects of the GG or GT genotype, whereas the L-PAFAH activity in the serum of GG subjects was significantly higher than that in the GT subjects $(\mathrm{P}<0,05)$.

PAFAH G994T polymorphism is associated with the clinical features of ARDS. PAF has been reported to be associated with the severity of ARDS and sepsis $(14,17)$. In addition, since the pro-inflammatory effect of PAF is abrogated by PAFAH, PAFAH activity has also been reported to affect the severity of ARDS. In the present study, the association between the polymorphism of PAFAH G994T and clinical features of ARDS, including the $\mathrm{PaO}_{2} / \mathrm{FiO}_{2}$ ratio, PEEP and lactic acid levels, as well as the SOFA, CPIS and APACHE II scores, was determined. As presented in Fig. 4, the $\mathrm{PaO}_{2} / \mathrm{FiO}_{2}$ ratio in GG subjects was significantly higher than that in GT subjects ( $\mathrm{P}<0.05$; Fig. 4A), whereas the PEEP (Fig. 4B) and lactic acid levels (Fig. 4C) in GG subjects were significantly lower than those in the GT subjects $(\mathrm{P}<0.05)$. In addition, the SOFA (Fig. 5A), CPIS (Fig. 5B) and APACHE II scores
(Fig. 5C) in GG subjects were lower as compared with those in GT subjects $(\mathrm{P}<0.05)$.

Effect of PAFAH G994T polymorphism on the prognosis of $A R D S$. The effect of the PAFAH G994T polymorphism on the survival rate of ARDS patients within the first 30 days following diagnosis was determined using Kaplan-Meier plots and the log-rank test. The 112 ARDS subjects were divided into two groups according to their LUS scores, with 56 subjects assigned to the group of high LUS scores (above the median) and the other 56 subjects assigned to the group of low LUS scores (below the median). The survival rates in the two groups were determined and compared. As presented in Fig. 6A, the subjects with a high LUS score exhibited a significantly higher rate of survival than those with a low LUS score $(\mathrm{P}<0.05)$. In addition, the 112 subjects were divided into two groups based on their genotype: GG subjects $(n=90)$ and GT subjects $(n=22)$. As presented in Fig. 6B, the GG genotype was associated with a significantly lower risk of mortality than the GT genotype $(\mathrm{P}<0.05)$. Subsequently, the 112 subjects were divided into four groups according to the combination of their genotypes and LUS scores: GG genotype and a high LUS score $(n=45)$, GT genotype and a high LUS score ( $\mathrm{n}=11)$, GG genotype and a low LUS score $(n=45)$ and GT genotype and a low LUS score $(\mathrm{n}=11)$. The prognosis of the subjects was compared between these four groups. As presented in Fig. 7, compared with the GT subjects with a low LUS score who presented the highest 

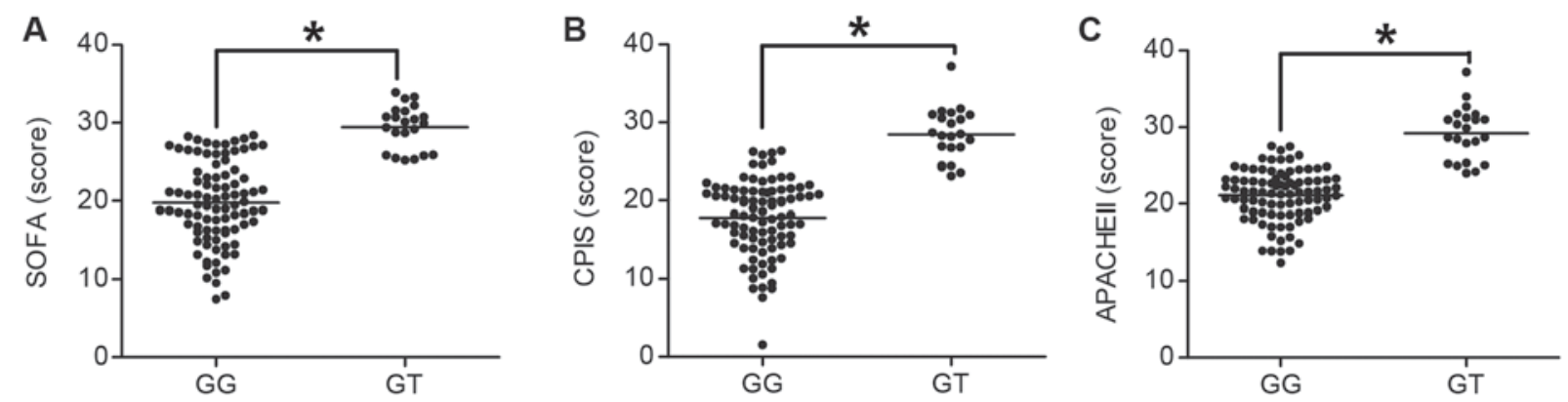

Figure 5. The platelet-activating factor acetylhydrolase G994Tpolymorphism is associated with the SOFA, CPIS and APACHE II score in patients with acute respiratory distress syndrome. The (A) SOFA, (B) CPIS and (C) APACHE II score in the GG group were lower than those in the GG group. "P<0.05. SOFA, sequential organ failure assessment; CPIS, clinical pulmonary infection score.
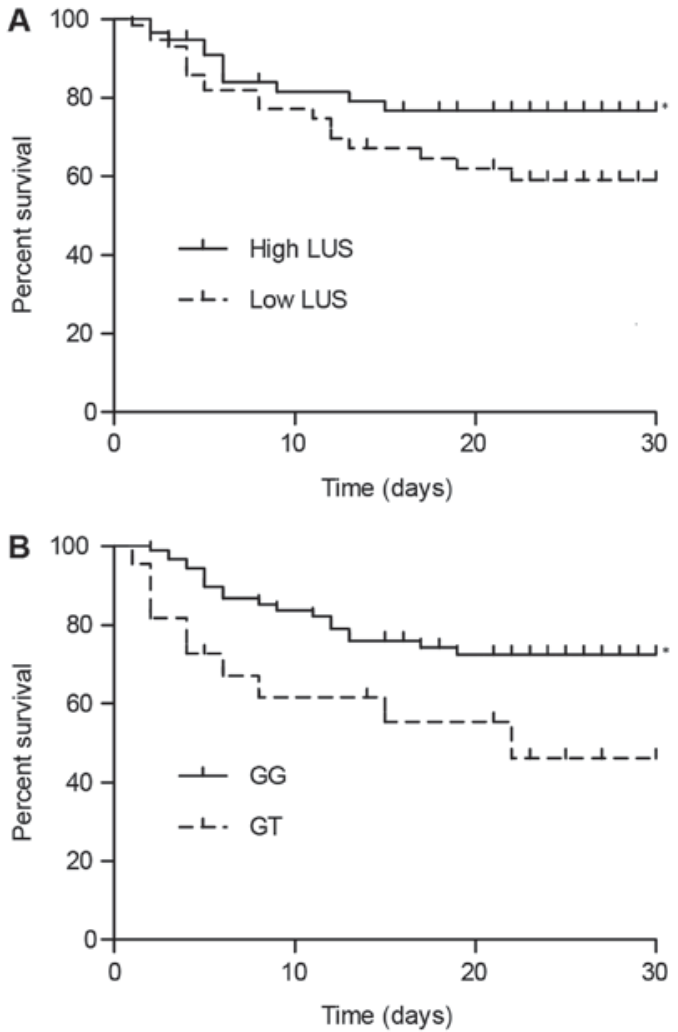

Figure 6. Clinical outcome for patients with acute respiratory distress syndrome is associated with platelet-activating factor acetylhydrolase G994T polymorphism genotypes and LUS score. (A) Subjects with a high LUS score exhibited a significantly higher survival rate than those with a low LUS score. (B) Subjects of the GG genotype had a significantly lower risk of death than those of the GT genotype. "P<0.05 vs. low LUS score or GT group. LUS, lung ultrasound.

mortality risk, the GG subjects with a high LUS score were had the lowest risk of mortality. In addition, the survival rates of GT subjects with a high LUS score and GG subjects with a low LUS score were significantly higher than that of GT subjects with a low LUS score. Furthermore, the survival rate among GT subjects with a high LUS score was significantly higher than that among GG subjects with a low LUS score $(\mathrm{P}<0.05)$.

\section{Discussion}

Tsubo et al (23) have used the LUS score to evaluate PEEP-induced changes in lung function. Other studies have

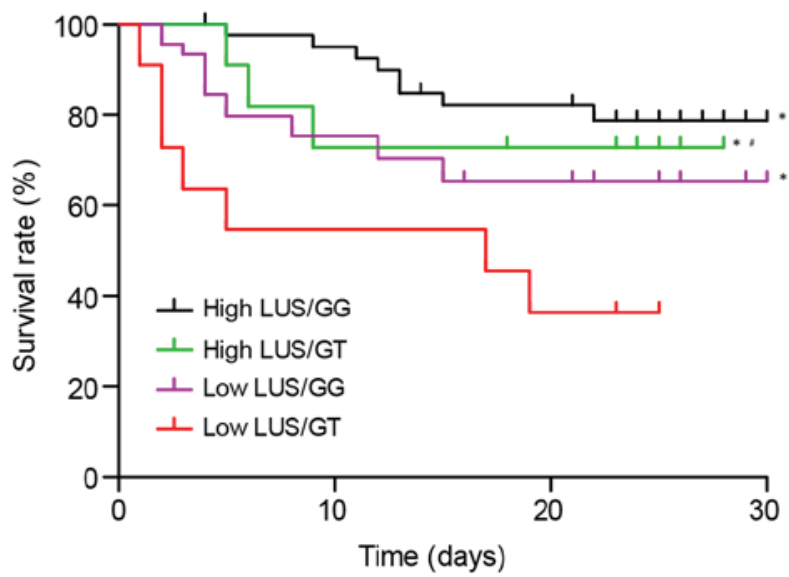

Figure 7. Patients with acute respiratory distress syndrome of the GG genotype and with a high LUS score had the lowest risk of death, while those of the GT genotype and with a low LUS score had the highest risk of death among the four groups. Furthermore, the survival rates of patients of the GT genotype with a high LUS score and the GG genotype with a low LUS score were higher than that of patients of GT genotype with a low LUS score. Accordingly, the survival rate of patients of the GT genotype and with a high LUS score was higher than that of patients of the GG genotype and with a low LUS score. ${ }^{*} \mathrm{P}<0.05$ vs. Low LUS/GT group; ${ }^{\text {}} \mathrm{P}<0.05$ vs. Low LUS/GG group. LUS, lung ultrasound.

reported that the LUS score accurately reflects changes in patients with heart diseases, as well as in pigs with oleic acid-induced acute lung injury $(24,25)$. In a study on ARDS patients, Bataille et al (26) determined that the B-line score of lung ultrasound (with a total score of 20 points) was able to predict the severity of the disease, with a score of 5 indicating mild disease, a score of 9 indicating moderate disease and a score of 11 indicating severe disease. Leblanc et al (27) reported that the LUS score was able to predict the incidence of ARDS in patients with pulmonary contusion with a sensitivity and specificity of 58 and $96 \%$, respectively. In addition, the LUS score in conjunction with the clinical features was able to predict the prognosis of ARDS with a higher accuracy than X-ray alone. Therefore, in the present study, LUS was performed to record 12 sections in the lung, with a total LUS score of 36 , to evaluate pulmonary fluid and pulmonary ventilation. The application of the LUS score in assessing the incidence of respiratory distress was first performed by Soummer et al (28), and their results indicated that the 
risk of extubation failure was low in patients with a LUS score of $<13$ at the end of the spontaneous breathing trial, whereas the risk of extubation failure was high in patients with a LUS score of $>17$. Caltabeloti et al (29) indicated that the bedside LUS score may be used to assess the change in pulmonary ventilation during ARDS and early septic shock, which occurred prior to the change in the oxygenation index. In the present study, negative correlations between the LUS score and the $\mathrm{PaO}_{2} / \mathrm{FiO}_{2}, \mathrm{PEEP}$ and lactic acid were identified, with correlation coefficients of $-0.493,-0.548$ and -0.642 , respectively. In addition, a negative correlation between the LUS and the SOFA, CPIS and APACHE II score was determined, with the correlation coefficients being $-0.598,-0.566$ and -0.567 , respectively.

As a $\mathrm{Ca}^{2+}$-independent catalyst of serine-dependent phospholipid hydrolysis, PAFAH belongs to the phospholipase A2 (PLA2) superfamily (30). Also known as lipoprotein-associated PLA2, the plasma form of PAFAH is a single 45-kilodalton polypeptide of 441 amino acids encoded by the PLA2G7 gene (31). Since PAFAH oxidizes phospholipids and hydrolyses PAF with a modified short fatty acyl chain esterified at the site of sn-2, it has a critical role in numerous physiological disorders, including ARDS, rheumatic diseases and severe anaphylaxis (32-35). It has been demonstrated that the activity of plasma PAFAH was significantly lower in ARDS patients carrying the His92 allele (16). This was surprising, given that the kinetics of the His92 mutant enzyme were not different from those of the wild-type PAFAH, although recombinant PAFAH proteins Val379 and Thr198 exhibited a decreased substrate affinity (i.e., a higher Michaelis Menten constant) (36). In addition, it has been indicated that non-survivors of ARDS had a significantly lower level of PAFAH circulating in their blood within $72 \mathrm{~h}$ of diagnosis $(16,37)$. Multiple studies have also indicated a correlation between decreased levels of PAFAH and an increased severity of inflammatory disorders. For instance, a decreased level of plasma PAFAH was reported to be associated with an enhanced severity of paediatric asthma (38), while a variation in the activity of plasma PAFAH at the onset of ARDS was demonstrated to be associated with a genetic alteration at the PAFAH locus (16-18).

Stafforini et al (15) identified the molecular structure of the human PAFAH gene, and a loss of its enzymatic activity was attributed to the G994T single-point mutation in its exon 9. The enzymatic activity of PAFAH is decreased in patients harbouring the heterozygous mutation, while it is fully abrogated in patients harbouring the homozygous mutation. Earlier studies have also identified that the polymorphism in the PAFAH gene was associated with the severity of certain atherosclerotic diseases, including peripheral artery occlusive disease, stroke, nephrotic syndrome in children and asthma attack (16-18). In the present study, the association between serum PAFAH activity and the PAHAF G994T SNP was investigated, and it was revealed that the activity of PAFAH and H-PAFAH in GG subjects was similar, whereas a higher L-PAFAH activity was observed in GG subjects. In addition, it was identified that the level of PEEP and lactic acid, as well as the SOFA, CPIS and APACHE II scores, in GG subjects was significantly lower than that in GT subjects, whereas the $\mathrm{PaO}_{2} / \mathrm{FiO}_{2}$ in $\mathrm{GG}$ subjects was significantly higher. In line with these results, it was also revealed that the genotype of the PAFAH G994T polymorphism and the LUS score were associated with the clinical outcomes of ARDS patients. The observation that the G994T polymorphism only influenced the L-PAFAH activity is different from the result of a previous study, which reported that the polymorphism influenced the PAHAF, H-PAHAF and D-PAHAF (16). This discrepancy may be attributed to the fact that differences in disease background, as the participants enrolled were either patients with polycystic ovary syndrome or healthy subjects. Further large-scale studies are required to confirm the results of the present study. The current study is however limited by a relatively small sample size. Further study with a larger sample size using more sophisticated statistical analysis is therefore warranted.

In conclusion, the present study was the first, to the best of our knowledge, to report that the G994T polymorphism in the PAFAH gene is associated with the clinical outcome of ARDS. Since this polymorphism was indicated to be associated with the activity of PAFAH and the prognosis of ARDS, which may contribute to the pathogenesis of ARDS, its functions should be further explored in order to develop novel therapeutic approaches to treat ARDS.

\section{Acknowledgements}

Not applicable.

\section{Funding}

The present study was financially supported by The funding of Medical Research of Wenzhou (grant no. 2017B04 from 2017).

\section{Availability of data and materials}

The datasets used and/or analysed during the current study are available from the corresponding author on reasonable request.

\section{Authors' contributions}

WL and RG designed the current study. SW, LW and ZW collected the literature and data, and analysed the data. YJ and XC interpreted and visualized the data. WL, SW and YJ prepared the manuscript. RG revised the final manuscript. All authors read and approved the final manuscript.

\section{Ethical approval and consent to participate}

The protocol of the present study was approved by the Ethics Committee of Wenzhou People's Hospital (Wenzhou, China) and written informed consent was obtained from all participants prior to the study.

\section{Patient consent for publication}

Not applicable.

\section{Competing interests}

The authors declare that they have no competing interests. 


\section{References}

1. Ware LB and Matthay MA: The acute respiratory distress syndrome. N Engl J Med 342: 1334-1349, 2000.

2. Rubenfeld GD, Caldwell E, Peabody E, Weaver J, Martin DP, Neff M, Stern EJ and Hudson LD: Incidence and outcomes of acute lung injury. N Engl J Med 353: 1685-1693, 2005.

3. Hogg JC: Neutrophil kinetics and lung injury. Physiol Rev 67 1249-1295, 1987.

4. Yoshida K, Kondo R, Wang Q and Doerschuk CM: Neutrophil cytoskeletal rearrangements during capillary sequestration in bacterial pneumonia in rats. Am J Respir Crit Care Med 174: 689-698, 2006.

5. Irish Critical Care Trials Group: Acute lung injury and the acute respiratory distress syndrome in Ireland: A prospective audit of epidemiology and management. Crit Care 12: R30, 2008.

6. Volpicelli G: Lung sonography. J Ultrasound Med 32: 165-171, 2013.

7. Volpicelli G, Skurzak S, Boero E, Carpinteri G, Tengattini M, Stefanone V, Luberto L, Anile A, Cerutti E, Radeschi G and Frascisco MF: Lung ultrasound predicts well extravascular lung water but is of limited usefulness in the prediction of wedge pressure. Anesthesiology 121: 320-327, 2014.

8. Zhao Z, Jiang L, Xi X, Jiang Q, Zhu B, Wang M, Xing J and Zhang D: Prognostic value of extravascular lung water assessed with lung ultrasound score by chest sonography in patients with acute respiratory distress syndrome. BMC Pulm Med 15: 98 , 2015.

9. Walker A: Breast milk as the gold standard for protective nutrients. J Pediatr 156 (2 Suppl): S3-S7, 2010.

10. Caplan MS, Sun XM, Hseuh W and Hageman JR: Role of platelet activating factor and tumor necrosis factor-alpha in neonatal necrotizing enterocolitis. J Pediatr 116: 960-964, 1990.

11. Shimizu T, Honda Z, Nakamura M, Bito H and Izumi T: Platelet-activating factor receptor and signal transduction. Biochem Pharmacol 44: 1001-1008, 1992.

12. Stafforini DM, McIntyre TM, Zimmerman GA and Prescott SM: Platelet-activating factor acetylhydrolases. J Biol Chem 272: 17895-17898, 1997.

13. Stafforini DM, Carter ME, Zimmerman GA, McIntyre TM and Prescott SM: Lipoproteins alter the catalytic behavior of the platelet-activating factor acetylhydrolase in human plasma. Proc Natl Acad Sci USA 86: 2393-2397, 1989.

14. Graham RM, Stephens CJ, Silvester W, Leong LL, Sturm MJ and Taylor RR: Plasma degradation of platelet-activating factor in severely ill patients with clinical sepsis. Crit Care Med 22: 204-212, 1994.

15. Stafforini DM, Satoh K, Atkinson DL, Tjoelker LW, Eberhardt C, Yoshida H, Imaizumi T, Takamatsu S, Zimmerman GA, McIntyre TM, et al: Platelet-activating factor acetylhydrolase deficiency. A missense mutation near the active site of an anti-inflammatory phospholipase. J Clin Invest 97: 2784-2791, 1996.

16. Fan P, Liu HW, Wang XS, Zhang F, Song Q, Li Q, Wu HM and Bai H: Identification of the G994T polymorphism in exon 9 of plasma platelet-activating factor acetylhydrolase gene as a risk factor for polycystic ovary syndrome. Hum Reprod 25: $1288-1294,2010$

17. Li S, Stuart L, Zhang Y, Meduri GU, Umberger R and Yates CR: Inter-individual variability of plasma PAF-acetylhydrolase activity in ARDS patients and PAFAH genotype. J Clin Pharm Ther 34: 447-455, 2009.

18. Li L, Yang Q, Li L, Guan J, Liu Z, Han J, Chao Y, Wang Z and $\mathrm{Yu} X$ : The value of lung ultrasound score on evaluating clinical severity and prognosis in patients with acute respiratory distress syndrome. Zhonghua Wei Zhong Bing Ji Jiu Yi Xue 27: 579-584, 2015 (In Chinese).

19. Shen GQ, Abdullah KG and Wang QK: The TaqMan method for SNP genotyping. Methods Mol Biol 578: 293-306, 2009.

20. Knaus WA, Draper EA, Wagner DP and Zimmerman JE: APACHE II: A severity of disease classification system. Crit Care Med 13: 818-829, 1985.
21. Sachdev A, Chugh K, Sethi M, Gupta D, Wattal C and Menon G: Clinical pulmonary infection score to diagnose ventilator-associated pneumonia in children. Indian Pediatr 48: 939-954, 2011.

22. Vincent JL, Moreno R, Takala J, Willatts S, De Mendonça A Bruining H, Reinhart CK, Suter PM and Thijs LG: The SOFA (Sepsis-related Organ Failure Assessment) score to describe organ dysfunction/failure. On behalf of the working group on sepsis-related problems of the European society of intensive care medicine. Intensive Care Med 22: 707-710, 1996.

23. Tsubo T, Sakai I, Suzuki A, Okawa H, Ishihara H and Matsuki A Density detection in dependent left lung region using transesophageal echocardiography. Anesthesiology 94: 793-798, 2001.

24. Agricola E, Picano E, Oppizzi M, Pisani M, Meris A, Fragasso G and Margonato A: Assessment of stress-induced pulmonary interstitial edema by chest ultrasound during exercise echocardiography and its correlation with left ventricular function. J Am Soc Echocardiogr 19: 457-463, 2006.

25. Gargani L, Lionetti V, Di Cristofano C, Bevilacqua G, Recchia FA and Picano E: Early detection of acute lung injury uncoupled to hypoxemia in pigs using ultrasound lung comets. Crit Care Med 35: 2769-2774, 2007.

26. Bataille B, Rao G, Cocquet P, Mora M, Masson B, Ginot J, Silva S and Moussot PE: Accuracy of ultrasound B-lines score and E/Ea ratio to estimate extravascular lung water and its variations in patients with acute respiratory distress syndrome. J Clin Monit Comput 29: 169-176, 2015.

27. Leblanc D, Bouvet C, Degiovanni F, Nedelcu C, Bouhours G, Rineau E, Ridereau-Zins C, Beydon L and Lasocki S: Early lung ultrasonography predicts the occurrence of acute respiratory distress syndrome in blunt trauma patients. Intensive Care Med 40: 1468-1474, 2014.

28. Soummer A, Perbet S, Brisson H, Arbelot C, Constantin JM, Lu Q and Rouby JJ; Lung Ultrasound Study Group: Ultrasound assessment of lung aeration loss during a successful weaning trial predicts postextubation distress*. Crit Care Med 40: 2064-2072, 2012.

29. Caltabeloti F, Monsel A, Arbelot C, Brisson H, Lu Q, Gu WJ, Zhou GJ, Auler JO and Rouby JJ: Early fluid loading in acute respiratory distress syndrome with septic shock deteriorates lung aeration without impairing arterial oxygenation: A lung ultrasound observational study. Crit Care 18: R91, 2014.

30. Dennis EA: The growing phospholipase A2 superfamily of signal transduction enzymes. Trends Biochem Sci 22: 1-2, 1997.

31. Derewenda ZS and Ho YS: PAF-acetylhydrolases. Biochim Biophys Acta 1441: 229-236, 1999.

32. Arai $\mathrm{H}$ : Platelet-activating factor acetylhydrolase. Prostaglandins Other Lipid Mediat 68-69: 83-94, 2002.

33. Vadas P, Gold M, Perelman B, Liss GM, Lack G, Blyth T, Simons FE, Simons KJ, Cass D and Yeung J: Platelet-activating factor, PAF acetylhydrolase, and severe anaphylaxis. N Engl J Med 358: 28-35, 2008

34. Vergne P, Praloran V, Treves R and Denizot Y: Decreased levels of serum platelet-activating factor acetylhydrolase in patients with rheumatic diseases. Mediators Inflamm 6: 241-242, 1997.

35. Nakos G, Kitsiouli E, Hatzidaki E, Koulouras V, Touqui L and Lekka ME: Phospholipases A2 and platelet-activating-factor acetylhydrolase in patients with acute respiratory distress syndrome. Crit Care Med 33: 772-779, 2005.

36. Kruse S, Mao XQ, Heinzmann A, Blattmann S, Roberts MH, Braun S, Gao PS,Forster J,Kuehr J,Hopkin JM, et al: The Ile198Thr and Ala379Val variants of plasmatic PAF-acetylhydrolase impair catalytical activities and are associated with atopy and asthma. Am J Hum Genet 66: 1522-1530, 2000.

37. Claus RA, Russwurm S, Dohrn B, Bauer M and Lösche W Plasma platelet-activating factor acetylhydrolase activity in critically ill patients. Crit Care Med 33: 1416-1419, 2005.

38. Miwa M, Miyake T, Yamanaka T, Sugatani J, Suzuki Y, Sakata S, Araki Y and Matsumoto M: Characterization of serum platelet-activating factor (PAF) acetylhydrolase. Correlation between deficiency of serum PAF acetylhydrolase and respiratory symptoms in asthmatic children. J Clin Invest 82: 1983-1991, 1988. 